
internationales

vol. 34 - $n^{\circ} 1 \mid 2018$

Mouvements migratoires d'hier et d'aujourd'hui en Italie

\title{
Éditorial : Coexistence, imbrication et superposition des flux migratoires italiens
}

Editorial: Coexistence, Interweaving and Overlapping of Italian Migration Flows Editorial: Coexistencia, entrelazamiento y superposición de los flujos migratorios italianos

\section{Paola Corti et Adelina Miranda}

\section{(2) OpenEdition}

Journals

Édition électronique

URL : https://journals.openedition.org/remi/9903

DOI : 10.4000/remi.9903

ISSN : $1777-5418$

Traduction(s) :

Presentazione: Coesistenza, interconnessioni e sovrapposizione dei flussi migratori italiani - URL https://journals.openedition.org/remi/9933 [it]

Editorial: Coexistence, Interweaving and Overlapping of Italian Migration Flows - URL : https:// journals.openedition.org/remi/10428 [en]

\section{Éditeur}

Université de Poitiers

\section{Édition imprimée}

Date de publication : 1 avril 2018

Pagination : 21-28

ISBN : 979-10-90426-61-0

ISSN : 0765-0752

\section{Référence électronique}

Paola Corti et Adelina Miranda, «Éditorial : Coexistence, imbrication et superposition des flux migratoires italiens », Revue européenne des migrations internationales [En ligne], vol. 34 - $n^{\circ} 1$ | 2018, mis en ligne le 28 décembre 2018, consulté le 21 avril 2022. URL : http://journals.openedition.org/ remi/9903; DOI : https://doi.org/10.4000/remi.9903 


\section{Éditorial}

\section{Coexistence, imbrication et superposition des flux migratoires italiens}

\section{Paola Corti ${ }^{1}$ et Adelina Miranda ${ }^{2}$}

Au moment où I'Italie apparait de plus en plus présente sur le devant de la scène internationale en raison des politiques de refoulement des étrangers aux frontières nationales mises en acte par ses gouvernements successifs, ce dossier de la REMI propose d'opérer un retour critique sur I'histoire migratoire de ce pays. Nous partons du constat que les migrations sont devenues un sujet éminemment politique dans l'espace italien. L'arrivée des personnes qui traversent la Méditerranée est accompagnée par l'élaboration de différentes rhétoriques. Comme le montrent les discours de deux sujets collectifs opérant dans l'espace politico-médiatique italien - les églises chrétiennes et la Ligue du Nord -, la légitimité ou l'illégitimité de la présence des migrants oscillent désormais entre deux pôles : I'universalité de l'être humain et le rejet xénophobe et raciste (Miranda, 2018). En tout cas, la mise en visibilité du phénomène utilise une succession d'évènements dramatiques qui ont mis au centre de l'attention médiatique et politique certains lieux, comme l'île de Lampedusa qui a été proposée pour le prix de la paix et qui a fait l'objet de nombreux films ou documentaires comme Fuocammare, de Gianfranco Rosi, primé à Berlin en 2016.

Dans ce contexte, l'adoption d'un regard distancié est essentielle pour relativiser les analyses actuelles qui parfois dissimulent la complexité du phénomène. Cette posture tient compte du fait que les savoirs produits par l'Occident " ne sont plus les seuls à parler des cultures et à les écrire " (Saillant, Kilani et Graezer Bideau, 2011 : 13). L'émergence de nouveaux lieux du savoir questionnent les conditions historiques, économiques et culturelles qui orientent la pensée occidentale et contribuent à la construction d'une géopolitique de la connaissance qui redéfinit les centres et les périphéries à l'intérieur d'un espace épistémique mondialisé (Mignolo, 2001). Afin d'observer les dynamiques migratoires produites " par le centre, depuis le centre ", nous nous inspirons de cette position en la conjuguant avec la tradition épistémologique de De Martino (2002). Pour

1 Historienne, Professeure, Université deTurin, Département de philosophie et de sciences de I'éducation, Via S. Ottavio 20, 10124Turin, Italie ; paola.corti@unito.it 
cet anthropologue italien, l'exercice d'un " ethnocentrisme critique " constitue une prise de conscience des limites des catégories et des outils intellectuels utilisés par le monde occidental afin d'appréhender les possibilités historiques de l'être humain. La prise de conscience de ces limites nous invite à expérimenter des nouvelles approches, pistes et méthodologies de recherche.

Appliquée à l'étude des migrations italiennes, cette perspective débouche sur plusieurs considérations critiques. Les analyses des configurations migratoires italiennes exhortent les chercheur.e-s à développer de nouveaux outils interprétatifs aptes à examiner des phénomènes qui contredisent l'élaboration des cadres interprétatifs cohérents et linéaires (Fabian, 2000). Dans cet éditorial nous retiendrons deux thématiques : la première est relative à la coexistence de différentes formes migratoires et la deuxième rappelle les enjeux de la catégorisation des migrant.e.s. Elles nous permettront d'argumenter que l'Italie, de par sa longue histoire migratoire, se présente comme un cas paradigmatique, une sorte de laboratoire qui démontre l'insuffisance des paradigmes migratoires " classiques".

\section{Les différentes formes migratoires}

Avec l'arrivée des étrangers en Italie, nous avons assisté à un renouvèlement de la recherche sur les migrations qui était jusque-là centrée sur l'émigration. Le rôle exercé par les médias et la perception de l'immigration de la part de la population ont constitué un levier qui a ouvert le débat sur l'immigration, mais aussi sur l'émigration, comme le montrent les succès littéraires des histoires, récits et romans publiés sur ce thème (Colucci, 2007 ; Sanfilippo, 2015), ainsi que la création de nombreux musées municipaux, régionaux et nationaux tel que le Museo Nazionale dell'Emigrazione Italiana ${ }^{3}$. L'adoption de nouvelles approches théorique et méthodologique (Gozzini, 2005 ; Corti et Sanfilippo, 2009 ; Bonifazi, 2013) a permis aux études sur l'émigration de sortir du cadre académique dans lequel ces recherches ont été longtemps cantonnées. En effet, en raison de l'amnésie réitérée de la classe politique italienne, les recherches sur les migrations n'avaient jusque-là trouvé leur place ni dans l'histoire nationale ni dans la mémoire collective (Martellini, 2003 ; Corti, 2005 et 2013 ; Sanfilippo, 2015). Ce regain d'intérêt a engendré un foisonnement d'études. La publication d'un volume entièrement dédié aux migrations dans la Storia d'Italia a contribué à la reconnaissance du fait que l'émigration est, avec I'Unité de I'Italie, un des phénomènes les plus marquants de I'histoire du pays (Corti et Sanfilippo, 2009). Ces travaux ont également permis de mieux saisir le rôle que les migrations italiennes ont joué, l'importance profonde qu'elles recouvrent encore dans le contexte européen et méditerranéen, et le fait qu'elles aient ouvert une série d'interrogations critiques quant aux théories reposant sur une vision évolutionniste des migrations dans les migrations studies.

3 Ce musée est actuellement fermé en attendant une nouvelle collocation. II est situé dans le Vittoriano (monument dédié à Victor Emmanuel, premier roi d'Italie, à Rome), devenu le lieu-symbole au niveau national de cette histoire (Lombardi et Prencipe, 2009 ; Tirabassi, 2009). 
Les textes présentés ici se situent dans ce sillon et ils confirment que I'histoire des migrations italiennes est plurielle, agencée autour de la coexistence de formes migratoires différentes. Pendant très longtemps I'Italie a été considérée comme n'étant qu'un pays d'émigration qui après l'achèvement d'un cycle d'exode massif se serait transformé en pays d'immigration. Or, le cadre migratoire italien a été, et continu à être stratifié comme le met en évidence I'article de Matteo Sanfilippo. Cet auteur reconstruit I'histoire de la mobilité depuis I'Unité du pays jusqu'à nos jours et, comme dans les travaux réalisés à la fin du XXe siècle, il montre que l'entrecroisement entre différentes formes de mobilité - émigration et immigration, mobilités internes, circulations des émigrés entre différents pays - n'est pas inédit. Aujourd'hui comme hier, les Italiens continuent à partir à l'étranger (Tirabassi et Del Prà, 2014 ; Sanfilippo et Vignali, 2017) et, en même temps, tout comme les immigrés, ils sont pris dans un mouvement intense à l'intérieur de I'Italie.

Michele Colucci et Stefano Gallo resituent dans leur article les continuités existant entre les migrations internationales et internes en montrant leurs variabilités selon les périodes historiques et les acteurs impliqués. La note de recherche d'Anna Badino complète ce panorama en proposant une comparaison entre la mobilité depuis le Sud vers le Nord de I'Italie et l'immigration des Italiens à Marseille. Ces contributions permettent d'opérer deux observations. La première est que la réorganisation des relations sociales dans les sociétés d'arrivée suit les mêmes logiques tant à l'étranger qu'au nord de I'Italie. La deuxième est la place comparable que ces dynamiques migratoires occupent aussi dans les histoires migratoires de l'Espagne, de la Grèce et du Portugal. Les analyses consacrées à l'Italie confirment que les migrations sont un trait consubstantiel des sociétés du pourtour de la Méditerranée et la nécessité d'observer les différentes facettes de ce phénomène afin d'en restituer les évolutions et les permanences en tenant compte de la politisation de la thématique (Miranda, 2008a ; Schmoll, Thiollet et Wihtol de Wenden, 2015). Celle-ci n'est pas un fait nouveau comme le souligne la mise en perspective des contributions d'Augusta Molinari, d'Elena Ambrosetti et Angela Paparusso. Augusta Molinari revient sur le départ de navires chargés de migrants italiens qui se dirigeaient vers les Amériques. Elle nous rappelle à quel point cette image, symbole par excellence des émigrations, nous renvoie à des formes de violence qui, aujourd'hui comme hier, sont la conséquence des politiques migratoires et que le voyage en mer est une expérience en soi qui structure le devenir de chaque migrant.Toutefois, comme le remarquent Elena Ambrosetti et Angela Paparusso, la traversée de la Méditerranée est de plus en plus liée à la distinction entre les demandeurs d'asile et les immigrants économiques, et à une gestion des faits migratoires qui considère les migrations comme un problème sécuritaire.

Les recherches présentées dans ce dossier démontrent l'intérêt heuristique à varier la focale du regard historique sur les faits migratoires, mais également sur les diverses échelles spatio-politiques - locales, régionales, nationales et internationales -, tout en s'appropriant le niveau subjectif. Comme le met en exergue l'étude de Selenia Marabello et Bruno Riccio sur les migrants arrivés du Sénégal et du Ghana dans la région émilienne, I'analyse du vécu biographique est un support analytique indispensable afin d'appréhender l'articulation des processus économiques et politiques, comme l'ont démontré les théories des réseaux et du transnationalisme (Collinson, 1993 ; Tarrius, 1993 ; Miranda, 1996 ; 
Sassen, 1996 ; Vertovec, 2009). Cette approche qui s'appuie sur la combinaison des échelles géographique et historique invite à repenser les modèles interprétatifs, comme le montre le cas des Italiens en France.

L'actuel regain d'intérêt pour l'histoire de ce courant migratoire est relié à différents facteurs. En premier lieu, il faut considérer la présence croissante de la nouvelle mobilité italienne qui, de par ses caractéristiques socioculturelles, peut être considérée comme représentative du panorama plus large des migrations intra-européennes contemporaines (Sirna, 2005 ; Fassio, 2014 ; Mourlane, 2017 ; Pfisch et Schmoll, 2017). En deuxième lieu, il faut tenir compte de la diffusion d'initiatives publiques qui traduisent les changements de la perception de l'immigration en provenance de la péninsule auprès des différentes générations d'Italiens en France (Ghidina et Violle, 2014). La récupération d'une mémoire collective de la part des nouvelles générations se concrétise sous la forme de différentes initiatives locales et rencontres culturelles (Teulière, 2002 ; Mourlane, 2017) qui contribuent à déplacer l'intérêt du champ d'études de l'histoire vers le vécu (Teulières, 2011 ; Gastaut, 2012 ; Mourlane, 2015 ; Felici, 2017) et vers l'art (Guibal et Cogne, 2011 ; Mourlane et Regnard, 2013) 4 . En troisième lieu, en raison de sa longue durée et de son impact sur les réalités économique et culturelle françaises, ce courant migratoire peut être considéré comme "une sorte de résumé de l'histoire de l'immigration en France avec tous les aléas qu'elle réserve: des manifestations racistes, l'intolérance religieuse ou bien des apports artistiques, culturels et gastronomiques qui ont enrichi le quotidien des Français " (Stora, $2017:$ 9).

Les recherches confirment donc que les parcours des migrants italiens en France ont été et continuent à être moins linéaires que le modèle prôné par les vulgates assimilationnistes. Par conséquent, l'analyse de ce cas est heuristique afin de lire d'autres expériences migratoires et d'encourager des recherches ayant une visée comparative. De surcroît, ce cas conduit à deux questionnements. Si au niveau subjectif les chercheurs ont réussi à étudier l'émigration et l'immigration comme conjointement engendrées (Sayad, 1991), pourquoi alors continuent-ils à analyser d'une manière disjointe les diverses formes de migrations ? Le manque d'attention aux formes de coexistence, de superpositions et d'enchevêtrements des différentes formes de migrations (qu'elles soient dérivées de l'esclavage, du colonialisme, de l'urbanisation ou de l'industrialisation) contribue-t-il à une sorte de hiérarchisation des formes migratoires et à la naturalisation des différences?

\section{Les enjeux de la catégorisation des migrant-e-s}

La remise en cause de la césure entre émigration et immigration italiennes interroge les catégories utilisées afin d'étudier les deux phénomènes. L'adoption d'une approche historicisée comparative restitue la variabilité de ces catégories ainsi que les continuités et les discontinuités qui définissent leurs limites. Un rappel permet d'éclaircir la difficulté de la question : en Italie, le mot "émigré "

4 Par exemple en 2017, I'exposition Ciao Italia!, au Musée de I'Immigration de Paris (Mourlane et Païni, 2017), a été accompagnée de la conférence connexe, L'Italie pour bagage (juin 2017). 
a été utilisé pour définir tant les migrants internes qu'internationaux. Lors de la première grande enquête réalisée au cours des années 1960 dans les quartiers périphériques de Milan et Turin, Alasia et Montaldi (1960) ont décrit les conditions de vie des émigrés vivant dans les "Corées " ${ }^{5}$. Ces auteurs nous ont livré une description qui aujourd'hui peut paraître étonnante : parmi ces Italiens nombreux étaient des "clandestins $"^{6}$, travaillant notamment dans le secteur de la construction, où ils étaient exploités par les entrepreneurs locaux. C'est la même image restituée par les nombreux travaux sur les Italiens qui avaient traversé les frontières nationales. De leur côté, les Italiens installés à l'étranger ont bataillé pour que le COEMIT (Comitato dell'Emigrazione Italiana institué en 1985) soit renommé en COMITES (Comitato degli Italiani all'Estero) revendiquant ainsi leur mise en distance de la figure de l'émigré.

D'une manière plus générale, le cas italien met en évidence qu'aujourd'hui les concepts de migrant et de migration ont connu une sorte de banalisation qui semble invalider leur valeur scientifique, d'autant plus qu'ils ont été absorbés par la sphère politique. Les Italiens, qui ont pris conscience tardivement de l'importance de l'immigration, ont réagi par le déni ou la dramatisation, mais surtout en produisant une rupture du lien entre l'immigration et l'émigration. Ce processus est d'autant plus intéressant qu'il permet de souligner comment les migrations sont utilisées dans l'élaboration des processus d'identité et d'altérisation. En effet, les analyses scientifiques montrent que les figures de l'immigré et de l'émigré sont imbriquées, mais cet enchevêtrement ne doit pas cacher le fait que dans le sens commun la figure de l'immigré n'est pas le miroir de celle de l'émigré. Se considérer comme appartenant à un pays d'émigration n'est pas la même chose que se considérer comme appartenant à un pays d'immigration ; les échelles de valeurs, les représentations, les jugements qui accompagnent les deux catégories ne coïncident pas.

L'introduction dans les études sur les migrations du prisme des rapports sociaux confirme que les figures des émigrés et des immigrés incarnent différemment les processus de subalternité et de domination. La revisitation à travers la perspective du genre du cas des " ensablés " proposée par Fabienne Le Houérou montre comment les transgressions sexuelles commises par les Italiens reposaient sur une idéologie fasciste qui, louant la supériorité de I'homme blanc, a encouragé les fantasmes de domination des colons. De son côté, l'article d'Adelina Miranda donne à voir des continuités inattendues : les frontières sémantiques élaborées entre les Italien·ne-s et les immigré-e·s évoquent de plus en plus l'imbrication genre/race à partir de l'image du migrant homme/noir/sans-travail.

Les contributions présentées montrent que pour la compréhension de la variabilité des configurations migratoires et les articulations hiérarchisantes entre les différentes figures migrantes il est nécessaire d'opérer une contex-

5 Les "Corées " étaient les bidonvilles dans lesquels étaient installés les émigrés italiens ; ces lieux rappelaient les images de villes détruites lors de la guerre en Corée.

6 Ces émigrés de l'intérieur étaient dans la plupart des cas des "clandestins ", puisque la loi fasciste, qui défendait à toute personne n'ayant pas un travail régulier et déclaré de changer de domicile, étant encore valable. De ce fait, les émigrés internes à I'Italie ne pouvaient pas avoir un domicile sans un travail régulier, mais ils ne pouvaient pas avoir de travail régulier sans avoir préalablement un domicile. 
tualisation des mouvements de population dans l'espace et dans le temps. De plus, elles remettent en cause nos pratiques épistémologiques : pouvonsnous continuer à recourir à un terme unique pour étudier des situations qui se dévoilent comme étant de plus en plus dissemblables ? Une mise en perspective des textes proposés dans ce numéro attire l'attention sur le fait que les concepts de migrants et de migrations semblent de moins en moins théoriquement et méthodologiquement efficaces. À la lecture des contributions, nous constatons qu'une déclinaison des concepts de migrants s'est imposée dans les discours scientifiques : "nouveaux " et " anciens " migrants ; "clandestins ", " irréguliers " et " sans papiers " ; migrants qualifiés et expatriés ; réfugiés politiques, demandeurs d'asile ; travailleurs établis et travailleurs saisonniers, etc. La multiplication des typologies fait référence à des catégories statistiques qui ordonnent les migrantee.s à partir de leurs motivations et des modalités de départ et d'arrivée, mais également aux cadres juridiques, politiques et ordinaires. Leur usage nous rappelle que les limites entre ces catégories sont réversibles et instables, que les causes et les motivations s'enchevêtrement dans les parcours des migrant.e.s, parfois s'accordant, mais parfois rentrant en contradiction avec les politiques migratoires.

Les études sur le cas italien que nous avons présentées dans ce dossier suggèrent, dans le sillon des migrations studies, d'un côté qu'il s'agit de dépasser la distinction entre migrants et non migrants et, d'un autre côté, qu'il n'est pas possible de définir les champs migratoires à l'intérieur desquels se déplacent anciens et nouveaux protagonistes à travers la simple analyse des trajectoires territoriales. De même, il n'est plus concevable d'utiliser des catégories qui reflètent sur le plan sémantique des typologies utilisées par un discours politique qui les instrumentalisent.

\section{Références bibliographiques}

Alasia Franco e Montaldi Danilo (1960) Milano Corea. Inchiesta sugli immigrati, Milano, Feltrinelli, 328 p.

Bonifazi Corrado (2013) L'Italia delle migrazioni, Bologna, il Mulino, 299 p.

Collinson Sarah (1993) Europe and International Migration, London, Pinter, 189 p.

Colucci Michele (2007) Storia o memoria? L'emigrazione italiana tra ricerca storica, uso pubblico e valorizzazione culturale, Studi Emigrazione, 167, pp. 721-728.

Colucci Michele e Gallo Stefano (Eds.) (2017) In cattedra con la valigia, Gli insegnanti tra stabilizzazionee mobilità, Rapporto 2017 sulle migrazioni interne in Italia, Roma, Donzelli editore, $188 \mathrm{p}$.

Colucci Michele e Gallo Stefano (Eds.) (2016) Fare spazio. Rapporto 2016 sulle migrazioni interne in Italia, Roma, Donzelli editore, $170 \mathrm{p}$.

Colucci Michele e Gallo Stefano (Eds.) (2015) Tempo di cambiare. Rapporto 2015 sulle migrazioni interne in Italia, Roma, Donzelli editore, $172 \mathrm{p}$.

Colucci Michele e Gallo Stefano (Eds.) (2014) L'arte di spostarsi. Rapporto sulle migrazioni interne in Italia, Roma, Donzelli editore, $170 \mathrm{p}$.

Corti Paola (2013) Temi e problemi di storia delle migrazioni, Viterbo, Sette Città, 132 p. 
Corti Paola (2005) L'emigrazione italiana e la sua storiografia: quali prospettive?, Passato e presente, 64, pp. 89-95.

Corti Paola e Sanfilippo Matteo (2012) L'Italia e le migrazioni, Bari-Roma, Laterza, 183 p.

Corti Paola e Sanfilippo Matteo (Eds.) (2009) Storia d'Italia. Annali 24. Migrazioni, Torino, Einaudi, 804 p.

De Martino Ernesto (2002) La fine del mondo, Torino, Einaudi, 727 p.

Fabian Johannes (2000 [1983]) II tempo e gli altri, La politica del tempo in antropologia, Napoli, L'Ancora del Mediterraneo, 233 p.

Fassio Giulia (2014) L'Italia non basterebbe. Migrazioni e presenza italiana a Grenoble dal secondo dopoguerra, Roma, Cisu, 302 p.

Felici Isabelle (2017) Sur Brassens et les autres " enfants " d'Italiens, Montpellier, Presses Universitaires de la Méditerranée, $259 \mathrm{p}$.

Gastaut Yvan (Éds.) (2012) Terres et gens de frontières : le cas exemplaire des migrations dans I'espace frontalier des Alpes du Sud, XIXe et XXe siècle, Migrations Societé, 140, pp. 51-298.

Ghidina Jean-Igor et Violle Nicolas (Éds.) (2014) Récits de migration. En quête de nouveaux regards, Clermond-Ferrand, CELIS, $334 \mathrm{p}$.

Gozzini Giovanni (2005) Le migrazioni di ieri edi oggi, Una storia comparata, Milano, Bruno Mondadori, $195 \mathrm{p}$.

Guibal Jean et Cogne Olivier (Éds.) (2011) Un air d'Italie. La présence italienne en Isère, Isère, Éditions du Musée dauphinois, 208 p.

Martellini Amoreno (Ed.) (2003) Cinque domande sulla storiografia dell'emigrazione a Emilio Franzina e Ercole Sori, Storia e problemi contemporanei, 34, pp. 15-31.

Meridiana (2018) Immigrazione, 91, 269 p.

Mignolo Walter (2001) Géopolitique de la connaissance, colonialité du pouvoir et différence coloniale, Multitudes, 6 (3), pp. 56-71.

Miranda Adelina (2018) Contrastes d'images. Les usages culturels de la figure des migrants arrivant en Italie par la Méditerranée, Revue Science and Video, 7, [en ligne]. URL : http://scienceandvideo.mmsh.univ-aix.fr/numeros/7/Pages/02. aspx

Miranda Adelina (2008a) Introduzione, Studi Emigrazione, 172, pp. 771-786.

Miranda Adelina (2008b) Migrare al femminile. Dinamiche culturali e appartenenza di genere in situazioni migratorie, Milano, McGraw-Hill, 198 p.

Miranda Adelina (2007) Le donne italiane in emigrazione tra passato e presente, in Fondazione Migrantes, Rapporto Italiani nel mondo 2007, Roma, Edizioni Idos, pp. 133-144.

Miranda Adelina (1996) Migrants et non-migrants d'une communauté italienne, Paris, L'Harmattan, $187 \mathrm{p}$.

Mourlane Stéphane (2017) Retour de mémoire, in Stéphane Mourlane et Dominique Païni Eds., Ciao Italia. Un siècle d'immigration et de cultures italiennes en France, Paris, La Martinière, pp. 176-183. 
Mourlane Stéphane (Éd.) (2015) Les Italiens dans le Sud-Est de la France : nouvelles perspectives, Archivio storico dell'emigrazione italiana, 11, pp. 7-93.

Mourlane Stéphane et Païni Dominique (Éds.) (2017) Ciao Italia. Un siècle d'immigration et de cultures italiennes en France, Paris, La Martinière, 192 p.

Mourlane Stéphane et Regnard Céline (Éds.) (2013) Empreintes italiennes. Marseille et sa région, Lyon, Lieux Dits Éditions, 132 p.

Nicosia Alessandro e Prencipe Lorenzo (Eds.) (2009) Museo Nazionale dell'Emigrazione Italiana, Roma, Gangemi editore, $494 \mathrm{p}$.

Pfirsch Thomas et Schmoll Camille (2017) Une nouvelle vague d'immigration italienne en France, in Stéphane Mourlane et Dominique Païni Éds., Ciao Italia. Un siècle d'immigration et de cultures italiennes en France, Paris, La Martinière, pp. 179-181.

Saillant Francine, Kilani Mondher et Graezer Bideau Florence (Éds.) (2001) Manifeste de Lausanne. Pour une anthropologie non hégémonique, Montréal, Liber, $143 \mathrm{p}$.

Sanfilippo Matteo (2015) Nuovi problemi di storia delle migrazioni italiane, Viterbo, Edizioni Sette città. 206 p.

Sanfilippo Matteo e Vignali Luigi Maria (Éds.) (2017) La nuova emigrazione italiana, Studi Emigrazione, 207, pp. 355-510.

Sassen Sakia (1996) Migranten, Siedler, Flüchtlinge, von der Massenauswanderung zur Festung Europa, Frankfurt, FischerTascenbuch, 215 p.

Sayad Abdelmalek (1991) L'immigration ou les paradoxes de l'altérité, Bruxelles, De Boeck, $331 \mathrm{p}$.

Schmoll Camille, Thiollet Hélène et Wihtol deWenden Catherine (2015) Migrations en Méditerranée. Permanences et mutations à l'heure des révolutions et des crises, Paris, CNRS Éditions, 282 p.

Sirna Francesca (2005) Italiani in Francia: un'integrazione riuscita?, Studi Emigrazione, 160, pp. 786-805.

Stora Benjamin (2017) L'immigration italienne en France, in Stéphane Mourlane et Dominique Païni Eds., Ciao Italia. Un siècle d'immigration et de cultures italiennes en France, Paris, La Martinière, pp. 8-11.

Tarrius Alain (1993) Territoires circulatoires et espaces urbains : différentiation des groupes migrants, Les Annales de la recherche urbaine, 59-60, pp. 51-60.

Teulières Laure (Éd.) (2011) Italiens. 150 ans d'émigration italienne en France et ailleurs, Toulouse, Éditalie éditions, 500 p.

Teulières Laure (2002) Mémoires et représentations du temps de guerre, Studi Emigrazione, 146, pp. 400-414.

Tirabassi Maddalena (2009) I luoghi della memoria delle migrazioni, in Paola Corti e Matteo Sanfilippo Eds., Migrazioni, Storia d'Italia, Annale 24, Torino, Einaudi, pp. 709-724.

Tirabassi Maddalena e Del Prà Alvise (2014) La meglio Italia. Le mobilità italiane nel XXI secolo, Torino, Accademia University Press, 240 p.

Vertovec Steven (2009) Transnationalism, London, Routledge, 205 p. 